\title{
Precision of collective oscillations in complex dynamical systems with noise
}

\author{
Fumito Mori ${ }^{1, *}$ and Alexander S. Mikhailov ${ }^{1,2}$ \\ ${ }^{1}$ Department of Physical Chemistry, Fritz, Haber Institute of the Max Planck Society, Faradayweg 4-6, 14195 Berlin, Germany \\ ${ }^{2}$ Department of Mathematical and Life Sciences, Hiroshima University, 1-3-1 Kagamiyama, Higashi-Hiroshima, Hiroshima 739-8526, Japan
}

(Received 25 January 2016; revised manuscript received 25 April 2016; published 9 June 2016)

\begin{abstract}
Two kinds of oscillation precision are investigated for complex oscillatory dynamical systems under action of noise. The many-cycle precision determined by the variance of the times needed for a large number of cycles is closely related to diffusion of the global oscillation phase and provides an invariant property of a system. The single-cycle precision given by the variance in durations of single cycles is sensitive to the choice of an output variable and output checkpoint; it can be improved by an appropriate selection of them. A general analysis of the precision properties based on the Floquet perturbation theory is performed and analytical predictions are verified in numerical simulations of a model oscillatory genetic network.
\end{abstract}

DOI: 10.1103/PhysRevE.93.062206

\section{INTRODUCTION}

Complex dynamical systems with attractive limit cycles generate stable periodic oscillations and can operate as clocks $[1,2]$. When such systems are subject to noise, fluctuations develop and the clocks become imprecise. The ability to maintain high oscillation precision despite noise is an essential property of biological systems [3-6] and much attention is paid to understand how this property emerges. Recently, the problem has been addressed for networks of coupled phase oscillators under synchronization conditions [7]. It has been found that, under given noise intensity, variability of periods is determined by the architecture of a network through its Laplacian spectrum. Thus, network architectures leading to enhanced precision of collective oscillations could be considered and analyzed [7]. When a pair of coupled phase oscillators was investigated, it was furthermore found that the period variability depends on how the measurement of periods is performed [8].

There are, however, also important biological systems, such as, e.g., genetic networks, where individual elements are not oscillators, but collective oscillations are nonetheless taking place [9-12]. Oscillation precision in nonoscillator systems with noise depends on structural organization of such systems and it should be clarified what structure is required for precision enhancement. Previously, without time-dependent noise, investigations have shown that genetic clocks with largely varying oscillation periods [13] and with high robustness against structural perturbations [14] could be designed.

Generally, two kinds of oscillation precision are to be distinguished. In some applications, it is important that the total time needed to perform a given large number of oscillations does not fluctuate much (many-cycle precision). For example, some electric fish generate rapid oscillations of electric discharge, where frequencies between 500 and $501 \mathrm{~Hz}$ can be discriminated; i.e., the time for 500 oscillation cycles must be precise [15]. On the other hand, clocks can also be used as devices that set the rhythm and control some regularly repeated events, as found, e.g., in the heartbeat. Then it is important

\footnotetext{
*Present address: Theoretical Biology Laboratory, RIKEN, 2-1 Hirosawa, Wako 351-0198, Japan; fumito.mori@ riken.jp
}

that durations of individual cycles do not fluctuate much. Therefore, minimizing variations in the times of individual cycles is essential (single-cycle precision) in this latter case.

Thus, depending on the purpose, different precision properties need to be employed. The aim of the present study is to analytically and numerically investigate what controls and influences different kinds of oscillation precision in general complex dynamical systems with attractive limit cycles under the action of noise.

It should be noted that the effects of noise in systems with translational invariance in time or space have been previously discussed [1,16-20]. In bistable reaction-diffusion systems with translational invariance in space, it has been shown that, as a result of noise, the traveling front position diffusively wanders [16]. In limit-cycle systems, translations in time correspond to changes in the global, or collective, oscillation phase [1,17]. The application of noise leads to diffusion of such a phase and, generally, the mean oscillation period also becomes modified [18]. The effects of colored and non-Gaussian noises on global oscillation phase have also been investigated $[19,20]$. However, a detailed analysis of temporal precision aspects has not yet been performed.

In this paper, we derive general expressions for both kinds of oscillation precision. Our analysis is valid for any limit-cycle systems, including the networks and systems that do not consist of the elements with individual oscillatory dynamics. In the next section, definitions of different precision properties are introduced. The theory of oscillation precision in general oscillatory dynamical systems is constructed in Sec. III. An example of the application of this theory to a model oscillatory genetic system is given in Sec. IV. The paper ends with conclusions and a discussion of the obtained results.

\section{DEFINITIONS AND EXAMPLES OF OSCILLATION PRECISION}

Different definitions of the oscillation period may be applied. For instance, an interval between adjacent oscillation peaks can be considered as a period. Moreover, isochrons of the collective phase in limit-cycle systems can be employed to determine the beginning or end points for a period. When temporal precision is considered, a period should be defined 

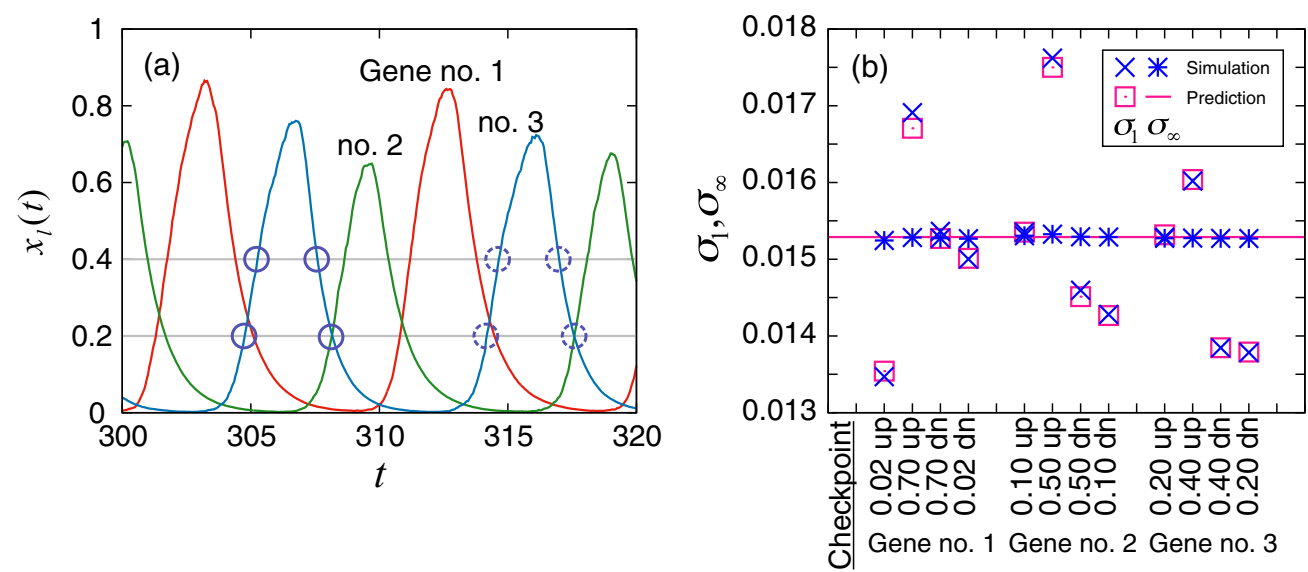

FIG. 1. (a) Stochastic oscillations of the expression levels of three genes in a model genetic network (see Sec. IV for details). The expression level of gene No. 3 is chosen as the output variable. To determine oscillation periods, two thresholds $(\bar{x}=0.2$ or $\bar{x}=0.4)$ can be used. The output variable can be checked to generate a spike for each cycle when it crosses a threshold in a certain direction (up or down). Thus, four checkpoints (shown by circles) are possible for a given output variable. (b) Precision properties of oscillations in the same genetic network. Along the horizontal axis, 12 different choices of checkpoints (outputs of three different genes with two choices of threshold levels for each gene and two passage directions, up or down). Cross symbols show single-cycle precision obtained from the stochastic oscillation data and boxes display the respective analytical predictions. Asterisks show 100-cycle precisions under the use of different checkpoints and the line indicates the analytical prediction for the many-cycle precision in the model.

by signals relevant for the function. In biological clocks, a series of spikes is typically produced and, in this case, the period is simply the time interval between the subsequent spikes. To generate spikes, a threshold mechanism is often employed. The spike is generated when the underlying continuous variable crosses a threshold for the first time in a certain cycle. In the present study, the spike-based definition for oscillation periods is chosen.

Suppose that some observable variables are persistently oscillating in a system. In genetic networks, for example, each such variable may correspond to the concentration of a protein expressed by a specific gene. We select one of the variables as the output $x_{l}(t)$ and introduce a certain threshold level $\bar{x}$. The spike timing of the $k$ th cycle, $t_{k}$, is defined as the time at which $x_{l}(t)$ passes $\bar{x}$ (in a specified direction, up or down) for the first time in this cycle; i.e., $t_{k}$ satisfies the conditions $x_{l}\left(t_{k}\right)=\bar{x}$ and $\left.\frac{d x_{l}}{d t}\right|_{t=t_{k}}>0$ (or $\left.\frac{d x_{l}}{d t}\right|_{t=t_{k}}<0$, if the spike is generated when it crosses the threshold in the down direction). Note that when this definition is applied, we exclude the events when the output variable crosses within a short time the threshold in both directions (see Appendix A). Such fluctuation events should not be counted as oscillation cycles.

The duration $T_{1, k}$ of the cycle $k$ is given by $T_{1, k} \equiv t_{k+1}-t_{k}$. We can also introduce the duration $T_{m, k}$ of $m$ consecutive cycles starting at cycle $m k$ that is given by $T_{m, k} \equiv t_{m(k+1)}-$ $t_{m k}$. The variance in durations of $m$ cycles is defined as

$$
V_{m}=E\left[\left(T_{m, k}-m \bar{T}\right)^{2}\right] .
$$

Here and below, $E[\cdots]$ always denotes both the average over a sequence of durations $k$ and the ensemble average over the realizations of a stochastic process. Because we only consider the regimes with steady oscillations and discard the transients, the two averages coincide. Moreover, the average single-oscillation period can be defined as $\bar{T}=E\left[T_{1, k}\right]$.
Through normalization of the variances, relative $m$-cycle precision $\sigma_{m}$ can be introduced as

$$
\sigma_{m}=\frac{1}{\bar{T}} \sqrt{\frac{V_{m}}{m}} .
$$

Specifically, single- and many-cycle precisions are

$$
\sigma_{1}=\frac{1}{\bar{T}} \sqrt{V_{1}}
$$

and

$$
\sigma_{\infty}=\lim _{m \rightarrow \infty} \frac{1}{\bar{T}} \sqrt{\frac{V_{m}}{m}} .
$$

As an example, Fig. 1(a) shows oscillations in the expression levels of three genes in a model genetic network introduced later in Sec. IV. The three genes forming the network are not identical and the expression level $x_{i}(t)$ of any of them $(i=1,2,3)$ can be chosen as the output variable $x_{l}(t)$. Here the expression level of gene No. 3 (blue) is chosen as the output variable. To determine oscillation periods, thresholds at $\bar{x}=0.20$ or 0.4 can be used. For each of the thresholds, the output variable can be checked to generate spikes when it crosses the threshold in either the up or the down direction. Thus, for a given output variable, four different choices of checkpoints exist. We also consider thresholds $\bar{x}=0.02$ or 0.70 for gene No. 1 and $\bar{x}=0.10$ or 0.50 for gene No. 2.

Using this example, we show some of the results in Fig. 1(b). Cross-shaped symbols in this figure display singlecycle precisions $\sigma_{1}$, defined for different output variables by using different checkpoints. It is seen that, by changing the output checkpoints and the output variables, precision properties may differ by up to around $30 \%$.

Hence, the single-cycle precision depends sensitively on the way signals or spikes are generated by oscillations. If, 
for example, our genetic circuit should work as a circadian clock with the period $\bar{T}=24 \mathrm{~h}$, the checkpoint-specific variation of single-cycle precision from 0.0135 to 0.0175 in Fig. 1(b) should correspond to an increase in the absolute period variation from 19.4 to $25.2 \mathrm{~min}$, which is an important difference.

Note that the single-cycle precision can be enhanced when a specific output variable and a particular checkpoint are selected (for example, by choosing gene No. 1 and the upward checkpoint at level $\bar{x}=0.02$ ). For comparison, numerical values of $\sigma_{100}$, approximately yielding the many-cycle precision, are also shown by asterisk symbols for different choices of output variables and checkpoints in Fig. 1(b). It is clearly seen that no significant variation of this property from one kind of measurement to another is present. Hence, it can provide an invariant characterization of oscillation precision. In the next section, a general analytical theory of precision properties is developed.

\section{ANALYTICAL EXPRESSIONS FOR DIFFERENT PRECISION PROPERTIES}

We consider a general $N$-dimensional dynamical system with noise described by

$$
\frac{d x}{d t}=f[x(t)]+\epsilon \boldsymbol{G}[\boldsymbol{x}(t)] \boldsymbol{\xi}(t),
$$

where $\boldsymbol{x}(t)$ is a state vector with $N$ components. The components of the noise vector $\boldsymbol{\xi}(t)$ are independent and identically distributed; they satisfy the conditions $E\left[\xi_{i}\right]=$ 0 and $E\left[\xi_{i}\left(t_{1}\right) \xi_{j}\left(t_{2}\right)\right]=\delta_{i j} \delta\left(t_{1}-t_{2}\right)$. The diagonal $N \times N$ matrix $\boldsymbol{G}$ depends on the state $\boldsymbol{x}(t)$. The coefficient $\epsilon$ is a small parameter $(\epsilon \ll 1)$. Equation (5) for $\epsilon=0$ is assumed to have a limit-cycle solution $\boldsymbol{x}(t)=\boldsymbol{p}(t)$ with a period $\tau$. The function $\boldsymbol{p}$ satisfies the equations $d \boldsymbol{p}(t) / d t=\boldsymbol{f}[\boldsymbol{p}(t)]$ and $\boldsymbol{p}(t)=\boldsymbol{p}(t+\tau)$. We assume $\boldsymbol{p}(0)=\boldsymbol{x}_{0}$.

When $\epsilon \neq 0$, stochastic oscillations are generated. We consider only statistically steady oscillations, which are realized after transient time. The output variable is the component $x_{l}$.

Our analysis will consist of three steps: linearization of dynamical equations, calculation of spatial variability based on the Floquet theory [21], and transformation from spatial to temporal variance.

Within one oscillation cycle, the solution $\boldsymbol{x}(t)$ of Eq. (5) can be written as

$$
\boldsymbol{x}(t)=\boldsymbol{p}(t)+\epsilon \boldsymbol{z}(t)+O\left(\epsilon^{2}\right),
$$

where $\|z(t)\| \ll \epsilon^{-1}$. The deviation $z(t)$ obeys the linearized equation,

$$
\frac{d z}{d t}=\boldsymbol{\Gamma}(t) z(t)+\boldsymbol{G}[\boldsymbol{p}(t)] \boldsymbol{\xi}(t)
$$

where $\boldsymbol{\Gamma}(t)$ is the Jacobian matrix, whose elements are given by

$$
\left.\Gamma_{i j}(t) \equiv \frac{\partial f_{i}}{\partial x_{j}}\right|_{\boldsymbol{x}(t)=\boldsymbol{p}(t)} .
$$

It has the periodicity $\Gamma(t+\tau)=\Gamma(t)$.
Here we focus on the unperturbed system

$$
\frac{d z}{d t}=\Gamma(t) z(t)
$$

and apply the Floquet theory to it. The solution $z(t)$ of Eq. (9) can be expressed as $\boldsymbol{z}(t)=\boldsymbol{U}(t) \boldsymbol{U}(0)^{-1} \boldsymbol{z}(0)$, where $\boldsymbol{U}(t)$ is the fundamental matrix solution, such that

$$
\boldsymbol{U}(t+\tau)=\boldsymbol{U}(t) \boldsymbol{U}(0)^{-1} \boldsymbol{U}(\tau)
$$

The matrix product $\boldsymbol{U}(0)^{-1} \boldsymbol{U}(\tau)$ is known as the monodromy matrix. We introduce furthermore the constant matrix $\boldsymbol{B}$ defined as

$$
\exp (\tau \boldsymbol{B}) \equiv \boldsymbol{U}(0)^{-1} \boldsymbol{U}(\tau)
$$

Then $z(t)$ can be rewritten as

$$
\boldsymbol{z}(t)=\boldsymbol{P}(t) \exp (t \boldsymbol{B}) \boldsymbol{U}(0)^{-1} \boldsymbol{z}(0),
$$

where

$$
\boldsymbol{P}(t) \equiv \boldsymbol{U}(t) \exp (-t \boldsymbol{B})
$$

Because of Eq. (10), the periodicity $\boldsymbol{P}(t+\tau)=\boldsymbol{P}(t)$ holds.

The right and left eigenvectors of $\boldsymbol{B}$ are defined as

$$
\begin{gathered}
\boldsymbol{B} \boldsymbol{\phi}_{i}=\lambda_{i} \boldsymbol{\phi}_{i}, \\
{ }^{t} \boldsymbol{\psi}_{i} \boldsymbol{B}=\lambda_{i}{ }^{t} \boldsymbol{\psi}_{i},
\end{gathered}
$$

where the superscript $t$ implies the transposition. Matrix $\boldsymbol{B}$ always has a zero eigenvalue $\lambda_{0}=0$. Moreover, the real parts of all of its eigenvalues should be nonpositive since the considered limit cycle is stable. We enumerate the eigenvalues in such a way that $\lambda_{0}=0 \geqslant \operatorname{Re}\left(\lambda_{1}\right) \geqslant \cdots \geqslant \operatorname{Re}\left(\lambda_{N-1}\right)$. The eigenvectors generally satisfy the biorthogonality relationship,

$$
{ }^{t} \boldsymbol{\psi}_{i} \boldsymbol{\phi}_{j}=\delta_{i j}
$$

where the eigenvectors are normalized as ${ }^{t} \boldsymbol{\psi}_{i} \boldsymbol{\phi}_{i}=1$. It can be easily verified that the right eigenvector corresponding to $\lambda_{0}=0$ is given by

$$
\boldsymbol{\phi}_{0}=\boldsymbol{U}(0)^{-1} \dot{\boldsymbol{p}}(0)
$$

Next, using the matrix $\boldsymbol{P}$, we introduce $\boldsymbol{y}(t)$ as

$$
\boldsymbol{y}(t) \equiv \boldsymbol{P}(t)^{-1} \boldsymbol{z}(t)
$$

so that Eq. (7) is transformed to

$$
\frac{d \boldsymbol{y}}{d t}=\boldsymbol{B} \boldsymbol{y}(t)+\boldsymbol{P}(t)^{-1} \boldsymbol{G}[\boldsymbol{p}(t)] \boldsymbol{\xi}(t),
$$

because $[\boldsymbol{\Gamma}(t) \boldsymbol{P}(t)-\dot{\boldsymbol{P}}(t)] \boldsymbol{y}(t)=\boldsymbol{B} \boldsymbol{y}(t)$. Equation (19) is a linear differential equation with constant coefficients and can be straightforwardly solved. We expand $\boldsymbol{y}(t)$ over the right eigenvectors of matrix $\boldsymbol{B}$ as $\boldsymbol{y}(t)=\sum_{j=0}^{N-1} c_{j}(t) \boldsymbol{\phi}_{j}$. Decomposition coefficients $c_{j}(t)$ obey stochastic differential 
equations

$$
\frac{d c_{j}(t)}{d t}=\lambda_{j} c_{j}(t)+{ }^{t} \boldsymbol{\psi}_{j} \boldsymbol{P}(t)^{-1} \boldsymbol{G}[\boldsymbol{p}(t)] \boldsymbol{\xi}(t),
$$

which can be integrated, yielding

$$
\begin{aligned}
c_{j}(t)= & \exp \left(\lambda_{j} t\right) \int_{0}^{t} \exp \left(-\lambda_{j} t^{\prime}\right){ }^{t} \boldsymbol{\psi}_{j} \boldsymbol{P}\left(t^{\prime}\right)^{-1} \boldsymbol{G}\left[\boldsymbol{p}\left(t^{\prime}\right)\right] \boldsymbol{\xi}\left(t^{\prime}\right) d t^{\prime} \\
& +\exp \left(\lambda_{j} t\right) c_{j}(0),
\end{aligned}
$$

where $c_{j}(0)$ is determined by the initial condition $\boldsymbol{x}(0)$.

Returning to Eq. (6) and expressing $\boldsymbol{x}(t)$ as the decomposition over right eigenvectors, we obtain

$$
\begin{aligned}
\boldsymbol{x}(t) & =\boldsymbol{p}(t)+\epsilon c_{0}(t) \dot{\boldsymbol{p}}(t)+\epsilon \boldsymbol{P}(t)\left\{\sum_{j \neq 0} c_{j}(t) \boldsymbol{\phi}_{j}\right\}+O\left(\epsilon^{2}\right) \\
& =\boldsymbol{p}\left[t+\epsilon c_{0}(t)\right]+\epsilon \sum_{j \neq 0} c_{j}(t) \boldsymbol{P}(t) \boldsymbol{\phi}_{j}+O\left(\epsilon^{2}\right)
\end{aligned}
$$

Equations (22) and (23) are valid as long as $\|\boldsymbol{z}(t)\| \ll \epsilon^{-1}$. According to them, the collective oscillation phase and the amplitude are $\Theta(t) \equiv t+\epsilon c_{0}(t)$ and $\boldsymbol{h}(t) \equiv \epsilon \sum_{j \neq 0} c_{j}(t) \boldsymbol{P}(t) \boldsymbol{\phi}_{j}$, respectively.

Note that we express the threshold level $\bar{x}$ for the output variable $x_{l}$ in terms of the limit-cycle trajectory satisfying $\boldsymbol{p}(0)=\boldsymbol{x}_{0}$ and the parameter $t_{\mathrm{cp}}$,

$$
\bar{x}=\left[\boldsymbol{p}\left(t_{\mathrm{cp}}\right)\right]_{l},
$$

where $[\boldsymbol{x}]_{l}$ represents the $l$ th element of the vector $\boldsymbol{x}$. The notation $[\boldsymbol{x}]_{l}$ is used to avoid confusion with the subscript representing eigenmodes. We emphasize that the nonstochastic parameter $t_{\mathrm{cp}}$ determines the threshold level $\bar{x}$ when the output variable $l$ is fixed.

To calculate the precision properties defined at $\bar{x}$, we consider an ensemble of trajectories satisfying the conditions that (i) $x_{l}$ passes (up or down) through checkpoint $\bar{x}$ at $t=t_{\mathrm{cp}}$ for the first time in the interval $0 \leqslant t_{\mathrm{cp}}<\tau$ and (ii) for the amplitude we have $E\left[\left|\boldsymbol{h}\left(t_{\mathrm{cp}}\right)\right|^{2}\right]=E\left[\left|\boldsymbol{h}\left(t_{\mathrm{cp}}+\tau\right)\right|^{2}\right]$ because oscillations are statistically steady. Condition (i) can be rewritten as $\left[x\left(t_{\mathrm{cp}}\right)\right]_{l}=\bar{x}$; furthermore, we have

$$
\left[\epsilon c_{0}\left(t_{\mathrm{cp}}\right) \dot{\boldsymbol{p}}\left(t_{\mathrm{cp}}\right)\right]_{l}=-\left[\epsilon \sum_{j \neq 0} c_{j}\left(t_{\mathrm{cp}}\right) \boldsymbol{P}\left(t_{\mathrm{cp}}\right) \boldsymbol{\phi}_{j}\right]_{l}+O\left(\epsilon^{2}\right) .
$$

Thus, the quantity $c_{0}\left(t_{\mathrm{cp}}\right)$ [or $c_{0}(0)$ in Eq. (21)] is determined by the left-hand side of Eq. (25). Condition (ii) leads to

$$
\begin{aligned}
& E\left[c_{j}(0) c_{k}(0)\right] \\
& =\frac{\exp \left[\left(\lambda_{j}+\lambda_{k}\right) \tau\right]}{1-\exp \left[\left(\lambda_{j}+\lambda_{k}\right) \tau\right]} \int_{0}^{\tau} \exp \left[-\left(\lambda_{j}+\lambda_{k}\right) t\right] \\
& \times{ }^{t} \boldsymbol{\psi}_{j} \boldsymbol{P}(t)^{-1} \boldsymbol{G}[\boldsymbol{p}(t)]^{2 t} \boldsymbol{P}(t)^{-1} \boldsymbol{\psi}_{k} d t,
\end{aligned}
$$

for $j, k=1, \ldots, N-1$. Hence, the initial distribution of $c_{j}(0)(j=0, \ldots, N-1)$ for the ensemble is determined by Eqs. (25) and (26).

\section{A. Single-cycle precision}

Equation (22) leads to

$$
\begin{aligned}
\boldsymbol{x}\left(t_{\mathrm{cp}}\right. & +\tau)-\boldsymbol{x}\left(t_{\mathrm{cp}}\right) \\
= & \epsilon\left[c_{0}\left(t_{\mathrm{cp}}+\tau\right)-c_{0}\left(t_{\mathrm{cp}}\right)\right] \dot{\boldsymbol{p}}\left(t_{\mathrm{cp}}\right) \\
& +\epsilon \sum_{j \neq 0}\left[c_{j}\left(t_{\mathrm{cp}}+\tau\right)-c_{j}\left(t_{\mathrm{cp}}\right)\right] \boldsymbol{P}\left(t_{\mathrm{cp}}\right) \boldsymbol{\phi}_{j}+O\left(\epsilon^{2}\right) \\
= & \epsilon \delta \Theta\left(t_{\mathrm{cp}}\right) \dot{\boldsymbol{p}}\left(t_{\mathrm{cp}}\right)+\epsilon \delta \boldsymbol{h}\left(t_{\mathrm{cp}}\right)+O\left(\epsilon^{2}\right)
\end{aligned}
$$

where $\epsilon \delta \Theta\left(t_{\mathrm{cp}}\right) \equiv \epsilon\left[c_{0}\left(t_{\mathrm{cp}}+\tau\right)-c_{0}\left(t_{\mathrm{cp}}\right)\right] \quad$ and $\quad \epsilon \delta \boldsymbol{h} \equiv$ $\epsilon \sum_{j \neq 0}\left[c_{j}\left(t_{\mathrm{cp}}+\tau\right)-c_{j}\left(t_{\mathrm{cp}}\right)\right] \boldsymbol{P}\left(t_{\mathrm{cp}}\right) \boldsymbol{\phi}_{j}$ represent the collective phase shift and the amplitude deviation after $\tau$, respectively.

The following relationships are further employed to transform from spatial to temporal variations:

$$
E\left[T_{1, k}-\tau\right]=E\left\{-\frac{1}{\left[\dot{\boldsymbol{p}}\left(t_{\mathrm{cp}}\right)\right]_{l}}\left[\boldsymbol{x}\left(t_{\mathrm{cp}}+\tau\right)-\boldsymbol{x}\left(t_{\mathrm{cp}}\right)\right]_{l}\right\}
$$

and

$$
E\left[\left(T_{1, k}-\tau\right)^{2}\right]=E\left\{\frac{1}{\left[\dot{\boldsymbol{p}}\left(t_{\mathrm{cp}}\right)\right]_{l}^{2}}\left[\boldsymbol{x}\left(t_{\mathrm{cp}}+\tau\right)-\boldsymbol{x}\left(t_{\mathrm{cp}}\right)\right]_{l}^{2}\right\} .
$$

These relationships approximately hold under the condition that the difference $\left|\boldsymbol{x}\left(t_{\mathrm{cp}}+\tau\right)-\boldsymbol{x}\left(t_{\mathrm{cp}}\right)\right|$ is small. Similar approximations employed for the transformation from the phase space to time have been numerically verified for the phase-oscillator systems [7,8].

Substitution of Eq. (27) into Eq. (28) yields $E\left[T_{1, k}\right]=\tau+$ $O\left(\epsilon^{2}\right)$. Thus, the average period of the weak-noise system is equal to that of the unperturbed system in our theory up to the order of $\epsilon$. This is consistent with the known fact that the average period is shifted by the higher-order quadratic term of about $\epsilon^{2}[18,19,22,23]$.

Substituting Eq. (27) and $E\left[T_{1, k}\right]=\tau$ into Eq. (29), we finally obtain the single-cycle precision, which is defined at threshold level $\bar{x}=\left[\boldsymbol{p}\left(t_{\mathrm{cp}}\right)\right]_{l}$ for output variable $x_{l}$, as

$$
\begin{aligned}
\sigma_{1}\left(t_{\mathrm{cp}}, l\right) & =\frac{1}{\tau} \sqrt{E\left(\left\{\epsilon \delta \Theta\left(t_{\mathrm{cp}}\right)+\frac{\epsilon\left[\delta \boldsymbol{h}\left(t_{\mathrm{cp}}\right)\right]_{l}}{\left[\dot{\boldsymbol{p}}\left(t_{\mathrm{cp}}\right)\right]_{l}}+O\left(\epsilon^{2}\right)\right\}^{2}\right)} \\
& =\frac{\epsilon}{\tau}\left[E\left[\delta \Theta^{2}\right]+E\left(\left\{\frac{\left[\delta \boldsymbol{h}\left(t_{\mathrm{cp}}\right)\right]_{l}}{\left[\dot{\boldsymbol{p}}\left(t_{\mathrm{cp}}\right)\right]_{l}}\right\}^{2}\right)+2 E\left\{\delta \Theta\left(t_{\mathrm{cp}}\right) \frac{\left[\delta \boldsymbol{h}\left(t_{\mathrm{cp}}\right)\right]_{l}}{\left[\dot{\boldsymbol{p}}\left(t_{\mathrm{cp}}\right)\right]_{l}}\right\}\right]^{1 / 2}+O\left(\epsilon^{3}\right),
\end{aligned}
$$


where

$$
\begin{aligned}
& E\left[\delta \Theta^{2}\right]=\int_{0}^{\tau} d t^{t} \boldsymbol{\psi}_{0} \boldsymbol{P}(t)^{-1} \boldsymbol{G}[\boldsymbol{p}(t)]^{2 t} \boldsymbol{P}(t)^{-1} \boldsymbol{\psi}_{0}, \\
& E\left(\left\{\frac{\left[\delta \boldsymbol{h}\left(t_{\mathrm{cp}}\right)\right]_{l}}{\left[\dot{\boldsymbol{p}}\left(t_{\mathrm{cp}}\right)\right]_{l}}\right\}^{2}\right)=\sum_{j=1}^{N-1} \sum_{k=1}^{N-1}\left[2-\exp \left(\lambda_{j} \tau\right)-\exp \left(\lambda_{k} \tau\right)\right] \frac{\left[\boldsymbol{P}\left(t_{\mathrm{cp}}\right) \boldsymbol{\phi}_{j}\right]_{l}\left[\boldsymbol{P}\left(t_{\mathrm{cp}}\right) \boldsymbol{\phi}_{k}\right]_{l}}{\left[\dot{\boldsymbol{p}}\left(t_{\mathrm{cp}}\right)\right]_{l}^{2}} \\
& \times \exp \left[\left(\lambda_{j}+\lambda_{k}\right) t_{\mathrm{cp}}\right]\left\{\int_{0}^{t_{\mathrm{cp}}} \exp \left[-\left(\lambda_{j}+\lambda_{k}\right) t\right]^{t} \boldsymbol{\psi}_{j} \boldsymbol{P}(t)^{-1} \boldsymbol{G}[\boldsymbol{p}(t)]^{2 t} \boldsymbol{P}(t)^{-1} \boldsymbol{\psi}_{k} d t\right. \\
& \left.+\frac{\exp \left[\left(\lambda_{j}+\lambda_{k}\right) \tau\right]}{1-\exp \left[\left(\lambda_{j}+\lambda_{k}\right) \tau\right]} \int_{0}^{\tau} \exp \left[-\left(\lambda_{j}+\lambda_{k}\right) t\right]^{t} \boldsymbol{\psi}_{j} \boldsymbol{P}(t)^{-1} \boldsymbol{G}[\boldsymbol{p}(t)]^{2 t} \boldsymbol{P}(t)^{-1} \boldsymbol{\psi}_{k} d t\right\},
\end{aligned}
$$

and

$$
E\left\{\delta \Theta\left(t_{\mathrm{cp}}\right) \frac{\left[\delta \boldsymbol{h}\left(t_{\mathrm{cp}}\right)\right]_{l}}{\left[\dot{\boldsymbol{p}}\left(t_{\mathrm{cp}}\right)\right]_{l}}\right\}=\sum_{j=1}^{N-1} \exp \left(\lambda_{j} \tau\right) \frac{\left[\boldsymbol{P}\left(t_{\mathrm{cp}}\right) \boldsymbol{\phi}_{j}\right]_{l}}{\left[\dot{\boldsymbol{p}}\left(t_{\mathrm{cp}}\right)\right]_{l}} \int_{0}^{\tau} \exp \left(-\lambda_{j} t\right)^{t} \boldsymbol{\psi}_{0} \boldsymbol{P}\left(t_{\mathrm{cp}}+t\right)^{-1} \boldsymbol{G}\left[\boldsymbol{p}\left(t_{\mathrm{cp}}+t\right)\right]^{2 t} \boldsymbol{P}\left(t_{\mathrm{cp}}+t\right)^{-1} \boldsymbol{\psi}_{j} d t
$$

We have used the relationship Eq. (26) to calculate Eq. (32). While Eq. (31) is independent of $t_{\mathrm{cp}}$ and $l$, Eqs. (32) and (33) are dependent on $l$ and periodic functions of $t_{\mathrm{cp}}$.

Thus, the single-cycle precision Eq. (30) consists of three terms of the order of $\epsilon$. The first term, corresponding to collective-phase diffusion, is independent of the choices of the checkpoint $t_{\mathrm{cp}}$ and the output variable $l$. The second term corresponds to the autocorrelation of amplitude deviations. Both these terms are positive. The third term in this expression represents cross correlation between the collective-phase shift and the amplitude deviation, and it is negative if these two variables are anticorrelated.

Because of this last term, the single-cycle precision can be sometimes better than the many-cycle precision corresponding to diffusion of the collective phase. We return to the discussion of this result in Sec. IV, where a specific example is examined.

\section{B. Many-cycle precision}

Equations (22) and (23) cannot be applied for calculation of $\boldsymbol{x}(t)$ at $t \rightarrow \infty$ because $c_{0}(t)$ can be then larger than $O\left(\epsilon^{-1}\right)$. To consider the dynamics over many cycles, we apply iteratively Eq. (23) for each next cycle, as explained below.

Starting points of consequent cycles are determined by the moments when the collective phase satisfies $\Theta(t)=k \tau$. Let $t=\tau-\Delta^{(1)}$ be the moment when $\Theta(t)=t+\epsilon c_{0}(t)=\tau$ for the first time. Then the stochastic time shift $\Delta^{(1)}$ is given by

$$
\Delta^{(1)}=\epsilon c_{0}\left(\tau-\Delta^{(1)}\right)=\epsilon c_{0}(\tau)+O\left(\epsilon^{2}\right) .
$$

Next we employ the following initial condition for the second cycle $t^{(2)} \equiv t-\left(\tau-\Delta^{(1)}\right)$ :

$$
\boldsymbol{x}\left(t^{(2)}=0\right)=\boldsymbol{p}(0)+\epsilon \sum_{j=1}^{N-1} c_{j}\left(\tau-\Delta^{(1)}\right) \boldsymbol{P}(0) \boldsymbol{\phi}_{0} .
$$

Under this condition, we have

$$
\boldsymbol{x}\left(t^{(2)}\right)=\boldsymbol{p}\left[t^{(2)}+\epsilon c_{0}^{(2)}\left(t^{(2)}\right)\right]+\epsilon \sum_{j=1}^{N-1} c_{j}^{(2)}\left(t^{(2)}\right) \boldsymbol{P}(0) \boldsymbol{\phi}_{0} .
$$

Because the collective phase for the second cycle is given by $\Theta^{(2)}=t^{(2)}+\epsilon c_{0}^{(2)}\left(t^{(2)}\right)$, we obtain $\Delta^{(2)}=\epsilon c_{0}^{(2)}(\tau)+O\left(\epsilon^{2}\right)$, where $\tau-\Delta^{(2)}$ denotes the time moment satisfying $\Theta^{(2)}=\tau$.
Thus, $\boldsymbol{x}\left(t^{(3)}=0\right)$ expresses the initial condition for the third cycle $t^{(3)} \equiv t^{(2)}-\left(\tau-\Delta^{(2)}\right)$. This operation can be iterated as

$$
\begin{aligned}
\boldsymbol{x}\left(t^{(m+1)}\right)= & \boldsymbol{p}\left[t^{(m+1)}+\epsilon c_{0}^{(m+1)}\left(t^{(m+1)}\right)\right] \\
& +\epsilon \sum_{j=1}^{N-1} c_{j}^{(m+1)}\left(t^{(m+1)}\right) \boldsymbol{P}(0) \boldsymbol{\phi}_{0},
\end{aligned}
$$

where

$$
\begin{gathered}
t^{(m+1)}=t-\left(m \tau-\sum_{k=1}^{m} \Delta^{(k)}\right) \\
\Delta^{(k)}=\epsilon c_{0}^{(k)}(\tau)+O\left(\epsilon^{2}\right) \\
c_{0}^{(k)}\left(t^{(k)}\right)=\int_{0}^{t^{(k)}}{ }^{t} \boldsymbol{\psi}_{j} \boldsymbol{P}(t)^{-1} \boldsymbol{G}[\boldsymbol{p}(t)] \xi\left(t-\sum_{k^{\prime}=1}^{k-1} \Delta^{\left(k^{\prime}\right)}\right) d t \\
c_{j(\neq 0)}^{(k)}\left(t^{(k)}\right)=\exp \left(\lambda_{j} t^{(k)}\right) \int_{0}^{t^{(k)}} \exp \left(-\lambda_{j} t\right)^{t} \boldsymbol{\psi}_{j} \\
\times \boldsymbol{P}(t)^{-1} \boldsymbol{G}[\boldsymbol{p}(t)] \boldsymbol{\xi}\left(t-\sum_{k=1}^{m-1} \Delta^{(k)}\right) d t \\
+\exp \left(\lambda_{j} t^{(k)}\right) c_{j}^{(k-1)}\left(\tau-\Delta^{(k-1)}\right)
\end{gathered}
$$

and $c_{j}^{(1)}(0)$ are determined by Eqs. (25) and (26).

Equation (37) determines $\boldsymbol{x}(t)$ for the duration of the $(m+1)$ th cycle. Within that cycle, the output variable will pass in the prescribed direction through the checkpoint $\bar{x}$ at $t^{(m+1)}=t_{\mathrm{cp}}^{(m+1)}$. Therefore, the total time $T_{m}$ between the first and the $(m+1)$ th passage through the checkpoint is

$$
\begin{aligned}
T_{m, 1}-m \tau & =t_{\mathrm{cp}}^{(m+1)}-t_{\mathrm{cp}}^{(1)}-\sum_{k=1}^{m} \Delta^{(k)} \\
& =t_{\mathrm{cp}}^{(m+1)}-t_{\mathrm{cp}}^{(1)}-\sum_{k=1}^{m}\left[\epsilon c_{0}^{(k)}(\tau)+O\left(\epsilon^{2}\right)\right] .
\end{aligned}
$$

Note that $t_{\mathrm{cp}}^{(m+1)}$ is independent of the course of the previous $m$ cycles and $t_{\mathrm{cp}}^{(1)}$ is a nonstochastic parameter. Therefore, such 
terms disappear from the many-cycle precision in the limit of an infinite number of cycles and we obtain

$$
\begin{aligned}
\sigma_{\infty} & =\frac{1}{\tau} \lim _{m \rightarrow \infty} \sqrt{\frac{1}{m}\left(\sum_{k=1}^{m}\left\{\epsilon^{2} E\left[c_{0}^{(k)}(\tau)^{2}\right]+O\left(\epsilon^{3}\right)\right\}\right)} \\
& =\frac{\epsilon}{\tau} \sqrt{E\left[\delta \Theta^{2}\right]}
\end{aligned}
$$

Thus, the many-cycle precision $\sigma_{\infty}$ corresponds to the collective-phase diffusion and is the same as the first term in Eq. (30). It is independent of the choices of the checkpoint and the output variable. Consequently, it represents an invariant property of the system.

\section{EXAMPLE: THE GENE EXPRESSION MODEL}

\section{A. Model}

As an example of a complex dynamical system with noise, we employ the gene expression model [24]. This model describes a network of genes that regulate the operation of one another. Each gene $i$ expresses a certain protein. The genes are connected into a network and, as we assume, can only inhibit the expression of the genes to which they are connected.

The model is given by a set of stochastic differential equations

$$
\begin{aligned}
\frac{d x_{i}}{d t}= & \frac{1}{1+\left(\kappa \sum_{j=1}^{N} A_{i j} x_{j}\right)^{n}}-a_{i} x_{i} \\
& +\frac{1}{\sqrt{U_{\max }}}\left[\frac{1+2 b}{1+\left(\kappa \sum_{j=1}^{N} A_{i j} x_{j}\right)^{n}}+a_{i} x_{i}\right]^{\frac{1}{2}} \xi_{i}(t) .
\end{aligned}
$$

Here $x_{i}$ represents dimensionless concentration of the protein expressed by gene $i$. The first term on the right side describes the process of protein generation (i.e., gene expression). This process is regulated (inhibited) by other genes. If $A_{i j}=1$, gene $j$ inhibits expression of gene $i$; otherwise (if $A_{i j}=0$ ), the regulation is absent. The parameter $\kappa$ specifies the intensity of gene regulation and $n$ is the Hill coefficient. The second term describes the process of protein degradation. We assume that degradation takes place at rates $a_{i}$, different for different genes. The last term takes into account the internal noise (see Ref. [24]). Such multiplicative noise arises because gene expression is a stochastic process where individual protein molecules are randomly produced (see Ref. [24] for detailed explanation). The intensity of such noise becomes larger when a smaller number $U_{\max }$ of proteins is produced by the gene. Noises for different genes are statistically independent, Gaussian, and $\delta$ correlated, so that we have $E\left[\xi_{i}(t)\right]=0$ and $E\left[\xi_{i}(t) \xi_{j}\left(t^{\prime}\right)\right]=\delta_{i j} \delta\left(t-t^{\prime}\right)$.

In our example, we choose the repressilator network [9] with three genes forming a ring: $N=3, A_{13}=A_{21}=A_{32}=$ 1 , and $A_{i j}=0$ for the others. The parameters are fixed as $n=3, \kappa=100, U_{\max }=50000, b=10, a_{1,2}=1.0$, and $a_{3}=$ 1.2. Note that because the degradation rates are different, the symmetry is broken and the dynamics of the three genes in the

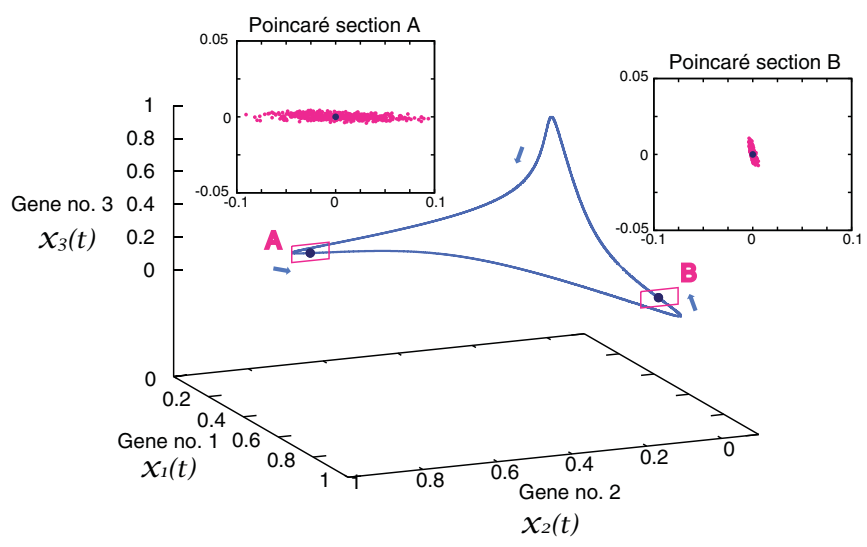

FIG. 2. The unperturbed limit cycle in the considered genetic network. Two small panels show Poincaré sections corresponding to checkpoints A ( $x_{1}=0.014$ upward $)$ and $\mathrm{B}\left(x_{1}=0.688\right.$ downward $)$, as obtained from stochastic simulations. In the panels, the origin of coordinates corresponds to the intersection point of the limit cycle. Note that statistical dispersion is larger at Poincaré section A than at Poincaré section B.

ring is not identical in the model. When noise is absent, the repressilator generates limit-cycle oscillations (Fig. 2).

\section{B. Simulation results}

Numerical simulations of stochastic equations (45) have been performed using the Heun method with a fixed time step of $10^{-3}$. The initial conditions $x_{i}(0)$ were randomly distributed in the interval $0<x_{i}(0)<1$. The observation of the times of $m$ cycles was started at $t=300$ and continued until the number of oscillations reached $10^{6}$. Threshold levels were chosen as $\bar{x}=0.02,0.70$ for gene No. $1, \bar{x}=0.10,0.50$ for gene No. 2 , and $\bar{x}=0.20,0.40$ for gene No. 3 .

Because of the fluctuations near the checkpoint, it can happen that the output variables crosses the threshold in both directions within a short time (Appendix A). Such pure fluctuation effects should not be treated as oscillation cycles. In the analytical theory, that was automatically taken into account because only cycle durations close to the oscillation period of the unperturbed system were considered. In numerical simulations, the following procedure was applied to exclude them: We installed additional threshold levels $\bar{x}=0.72$ and $\bar{x}=0.015$ for gene No. 1 . If noise is absent, the output $x_{1}$ crosses $\bar{x}=0.70$ up, $\bar{x}=0.72$ up, $\bar{x}=0.70$ down, $\bar{x}=0.02$ down, $\bar{x}=0.015$ down, and $\bar{x}=0.02$ up, in this order. We have applied this order to count crossing events; i.e., after passing a checkpoint, we do not check events that $x_{1}$ passes the checkpoint until $x_{1}$ goes through all the other checkpoints in this order. The thresholds $\bar{x}=0.52$ and $\bar{x}=0.05$ for gene No. 2 and $\bar{x}=0.45$ and $\bar{x}=0.15$ for gene No. 3 also had been employed for the same purpose.

An example of the oscillations observed in the repressilator model and the four checkpoints employed for gene No. 3 are shown in Fig. 1(a). Numerically determined values of singleand many-cycle precisions for different choices of output variables and checkpoints are displayed in Fig. 1(b). In addition, we show Poincaré sections corresponding to checkpoints A 

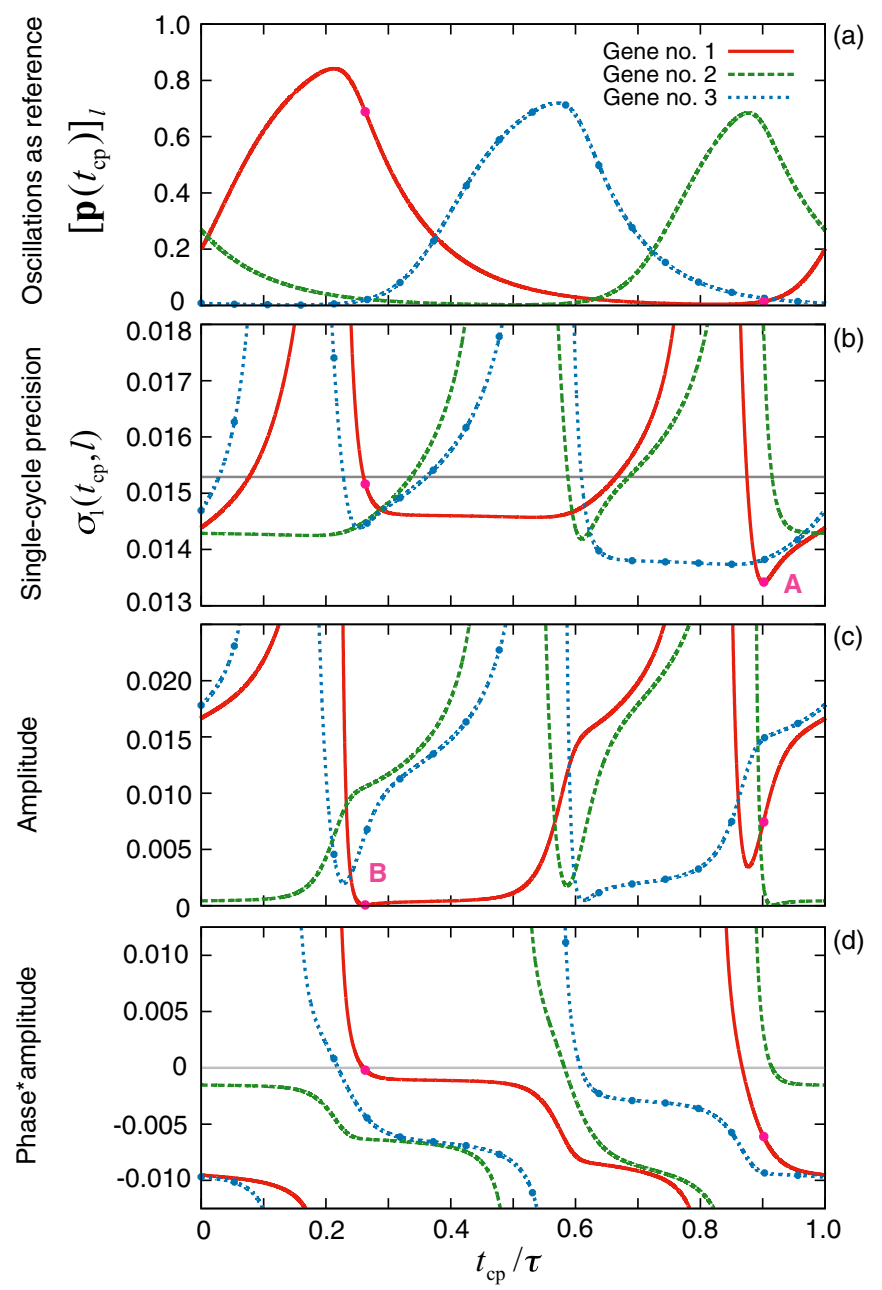

FIG. 3. Periodic properties predicted by the analytical theory for the considered genetic network, plotted as functions of the common parameter $t_{\mathrm{cp}}$. (a) Limit-cycle oscillations for each gene, $\left[\boldsymbol{p}\left(t_{\mathrm{cp}}\right)\right]_{l}$, for one cycle in absence of noise. These oscillations are used as the reference for the checkpoint threshold in panels (b)-(d) because the parameter $t_{\mathrm{cp}}$ fixes the threshold level through $\bar{x}=\left[\boldsymbol{p}\left(t_{\mathrm{cp}}\right)\right]_{l}$. (b) The single-cycle precision $\sigma_{1}$ given by Eq. (30). The straight line represents many-cycle precision $\sigma_{\infty}$ given by Eq. (44). (c) The amplitude variance is given by $\epsilon^{2} \times$ Eq. (32). (d) The cross correlation between collective-phase shift and amplitude deviation given by $\epsilon^{2} \times$ Eq. (33). A and B are the checkpoints with the minimum values of $\sigma_{1}$ and the minimum amplitude variance for gene No. 1. Red (solid), green (dashed) and blue (dotted) curves are for genes No. 1, No. 2, and No. 3, respectively.

( $x_{1}=0.014$ upward $)$ and B $\left(x_{1}=0.688\right.$ downward $)$ in Fig. 2 to visualize spatial fluctuations.

\section{Analytical predictions}

Both kinds of precision for the repressilator network could be estimated using analytical results Eq. (30) and (44). The limit-cycle trajectory $\boldsymbol{p}(t)$ was obtained through numerical integration of Eq. (5) with $\epsilon=0$ (Fig. 2). We assumed $\boldsymbol{p}(0)=\boldsymbol{x}(0)={ }^{t}(0.200,0.268,0.008)$. The limit-cycle oscillations $p_{i}(t)$ for one cycle in absence of noise are plotted as a function of time in Fig. 3(a). The unperturbed period was $\tau=9.413$. Then we numerically solved Eq. (9) for a few cycle periods, where the initial conditions were set to $\mathbf{z}_{a}(0)={ }^{t}(1,0,0), z_{b}(0)={ }^{t}(0,1,0)$, and $z_{c}(0)={ }^{t}(0,0,1)$, i.e., $\boldsymbol{U}(0)=\boldsymbol{I}$. The fundamental solutions $\mathbf{z}_{a}(t), \mathbf{z}_{b}(t)$, and $\mathbf{z}_{c}(t)$ are shown in Appendix B. The fundamental matrix solution was given by $\boldsymbol{U}(t)=\left[\mathbf{z}_{a}(t), \mathbf{z}_{b}(t), \mathbf{z}_{c}(t)\right]$.

Then the matrices of $\boldsymbol{B}$ and $\boldsymbol{P}$ were defined by $\exp (\tau \boldsymbol{B})=$ $\boldsymbol{U}(\tau)$ and $\boldsymbol{P}(t)=\boldsymbol{U}(t) \exp (-t \boldsymbol{B})$. We have checked that the periodicity $\boldsymbol{P}(t+\tau)=\boldsymbol{P}(t)$ holds. Numerical diagonalization of $\boldsymbol{B}$ provided two negative eigenvalues $\left(\lambda_{1,2}<0\right)$ and one (approximately) zero eigenvalue $\left(\lambda_{0}=0\right)$. Moreover, the eigenvectors $\boldsymbol{\psi}_{i}$ and $\boldsymbol{\phi}_{i}$ were obtained. There $\boldsymbol{\phi}_{i}$ and $\boldsymbol{\psi}_{j}$ were normalized so that these satisfy ${ }^{t} \boldsymbol{\psi}_{i} \boldsymbol{\phi}_{j}=\delta_{i j}$. We assumed $\boldsymbol{\phi}_{0}=\dot{\boldsymbol{p}}(0)$. In Appendix B, the matrix $\boldsymbol{B}$, the eigenvalues $\lambda_{i}$, eigenvectors $\boldsymbol{\psi}_{i}$ and $\boldsymbol{\phi}_{i}$, and internal noise intensity $\boldsymbol{G}[\boldsymbol{p}(t)]$ are given.

Putting these expressions into Eqs. (30)-(33) and (44), we have obtained the predicted values for $\sigma_{1}$ and $\sigma_{\infty}$. As shown in Fig. 1(b), these values given by our analytical expressions have agreed well with the numerical results for all choices of the output variables and checkpoints.

To analyze the checkpoint dependence, single-cycle precisions $\sigma_{1}\left(t_{\mathrm{cp}}, l\right)$ given by Eq. (30) for different output variables $l=1,2,3$ are plotted as functions of $t_{\mathrm{cp}}$ determining the threshold level $\bar{x}=\left[\boldsymbol{p}\left(t_{\mathrm{cp}}\right)\right]_{l}$ in Fig. 3(b). As we see by comparing Figs. 3(a) and 3(b), $\sigma_{1}$ is increased and precision becomes poor when the checkpoint is chosen near the maximum of the respective output variable. We do not show the dependence very close to the maxima because, due to fluctuations, the observables often do not pass then through the threshold in the next oscillation cycle.

There are, however, also time intervals where $\sigma_{1}$ is small and therefore the single-cycle precision is high. The minimum value of $\sigma_{1}$ is 0.0134 and it is reached at checkpoint $\mathrm{A}\left\{\left[\boldsymbol{p}\left(t_{\mathrm{cp}} / \tau=0.90\right)\right]_{1}=0.014\right\}$ if the expression of gene No. 1 is chosen as the output variable [Fig. 3(b)]. Hence, if our model network needs to be used as a rhythm generator, the best precision would be achieved if the output signal is sent from gene No. 1 at checkpoint A.

Moreover, we can note that the output variable that yields the smallest $\sigma_{1}$ is dependent on the timing $t_{\mathrm{cp}}$. For example, inside the time window $0 \leqslant t_{\mathrm{cp}} / \tau \leqslant 0.2$ in Fig. 3(b), gene No. 2 is the best one in the sense of the single-cycle precision. Thus, by changing the output variables as gene No. $2 \rightarrow$ No. $1 \rightarrow$ (No. 2 short interval) $\rightarrow$ No. $3 \rightarrow$ No. 1 , the clock can keep sending precise signals for the whole period. This mechanism might be employed in real circadian clocks because they have multiple variables for each function.

Next we can discuss the factors affecting the single-cycle precision. The standard deviation of amplitude observed at a Poincaré section, $E\left(\left\{\frac{\left[\delta \boldsymbol{h}\left(t_{\mathrm{cp}}\right)\right]_{l}}{\left[\dot{\boldsymbol{p}}\left(t_{\mathrm{cp}}\right)\right]_{l}}\right\}^{2}\right)$ given by Eq. (32), is shown in Fig. 3(c) (see also Fig. 2). At checkpoint B $\left\{\left[\boldsymbol{p}\left(t_{\mathrm{cp}} / \tau=\right.\right.\right.$ $\left.0.25)]_{1}=0.688\right\}$, the amplitude variance is minimal, i.e., the actual stochastic trajectories are close to the unperturbed limit cycle. It should be stressed that this point is however different from the best checkpoint (labeled A) for the single-cycle precision. This is because the cross correlation between the collective phase and the amplitude, $E\left\{\delta \Theta\left(t_{\mathrm{cp}}\right) \frac{\left[\delta \boldsymbol{h}\left(t_{\mathrm{cp}}\right)\right]_{l}}{\left[\dot{\boldsymbol{p}}\left(t_{\mathrm{cp}}\right)\right]_{l}}\right\}$ given by Eq. (33) plays an important role in determining the single-cycle 

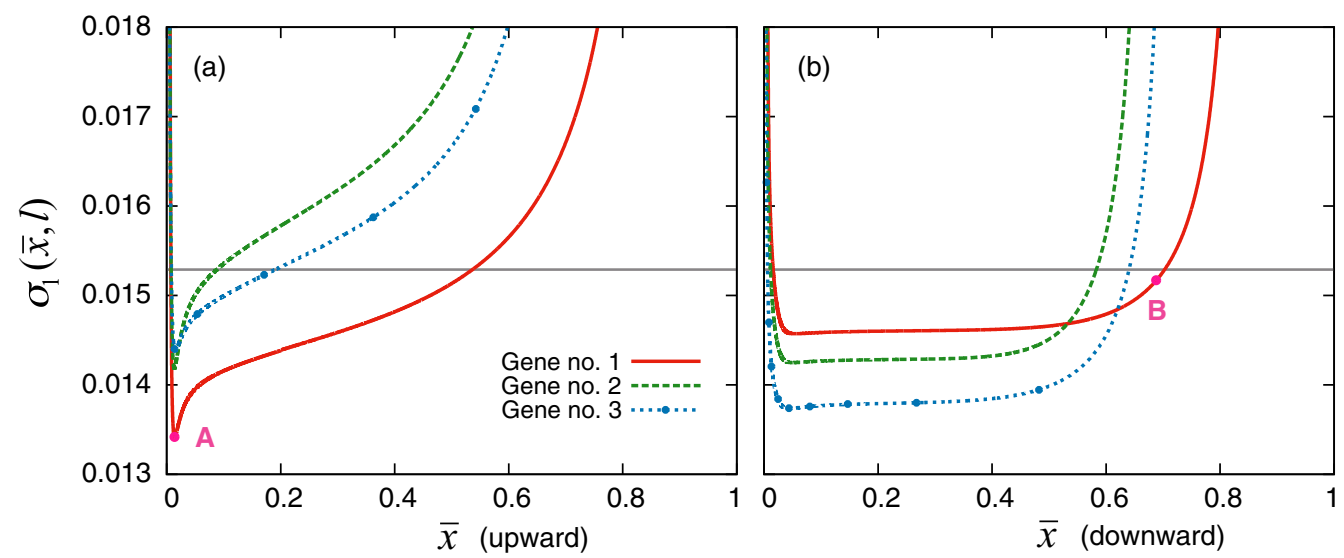

FIG. 4. Single-cycle precision as a function of threshold level $\bar{x}$, which output variable $x_{l}$ passes (a) upward and (b) downward. This is a rearrangement of Figs. 3(a) and 3(b). The straight line represents many-cycle precision. Checkpoints A and B correspond to those shown in Figs. 2 and 3.

precision. In Fig. 3(d), we can see that the absolute value of this correlation at checkpoint $\mathrm{A}$ is much larger than that at checkpoint B. Thus, the negative cross correlation improves the single-cycle precision at checkpoint A.

In Figs. 4(a) and 4(b), we also the show single-cycle precision as a function of threshold level $\bar{x}$, when $x_{l}$ crosses up and down, respectively. While gene No. 1 is the best choice for the single-cycle precision for the upward case (a), gene No. 3 has the minimum $\sigma_{1}$ in the wide range for the downward case (b). Therefore, if the system generates spikes only when the output variable crosses $\bar{x}$ down, gene No. 3 should be employed as the output variable yielding the best single-cycle precision.

\section{DISCUSSION AND CONCLUSIONS}

Approximate analytical expressions for single- and manycycle precisions in complex dynamical oscillatory systems with noise have been derived. Their validity has been numerically confirmed by using a genetic network model. Because the many-cycle precision is invariant, it can universally characterize the long-time behavior of the entire system. On the other hand, the single-cycle precision is sensitively dependent on the choice of a checkpoint and the output variable. While amplitude variance worsens the single-cycle precision, the cross correlation between the collective phase and the amplitude can improve it if such correlation is negative. Thus, statistical dispersion of stochastic trajectories and temporal precision have to be clearly distinguished.

Our results furthermore suggest the design principles of dynamical systems that should operate as clocks. Because the many-cycle precision cannot be improved only by changing the output method, the design of the dynamical system needs to be modified if one wants to have a clock with a better accuracy over a long time. On the other hand, an optimal choice of the output variable and the output timing yields the signals with high single-cycle precision, which should be taken if the system is employed to generate a rhythm. Moreover, by appropriately switching the output variables, it is possible to keep sending a precise signal within the whole cycle, as may be required for a circadian clock. It is an interesting and open question whether real biological systems employ such mechanisms of precision enhancement in their natural evolutionary design.

\section{ACKNOWLEDGMENTS}

Financial support from the Volkswagen Foundation (Germany) is gratefully acknowledged.

\section{APPENDIX A}

In this appendix, we analyze the statistics of threshold crossing events for the output variable. The analysis is performed using the example of the genetic network. We demonstrate that, at the considered levels of noise, full oscillation cycles can be always distinguished from the effects of rapid fluctuations in the output variable near the checkpoint.

Figure 5 shows the histogram of time intervals between next threshold crossing events for the genetic model [Eq. (45)] with the output variable $x_{1}$ and the threshold $\bar{x}=0.02$, when checking is performed if the variable crosses the threshold in

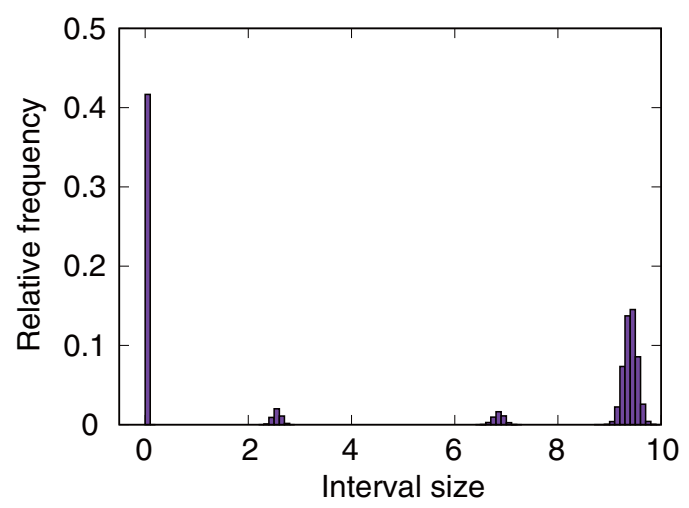

FIG. 5. Distribution of time intervals between the next time moments when the output variable $x_{1}(t)$ crosses the threshold level $\bar{x}=0.02$ in the upward direction for the model genetic system Eq. (45). 
the upward direction. As can be seen, the histogram consists of four distinct groups.

Full oscillation cycles, which should be used when oscillation precision is estimated, are only in the group with the largest time intervals. The shortest time intervals correspond to the events when, due to noise, the output variable crosses the threshold up, then down, and then again up within a short time. Such fluctuation events should not be counted as oscillation cycles. Additionally, there are two small groups with the centers near 2.5 and 7 . They are due to the events when the output variable goes down, but, due to noise, also crosses the threshold in the up direction, before continuing to go down. Such events also do not correspond to oscillation cycles.

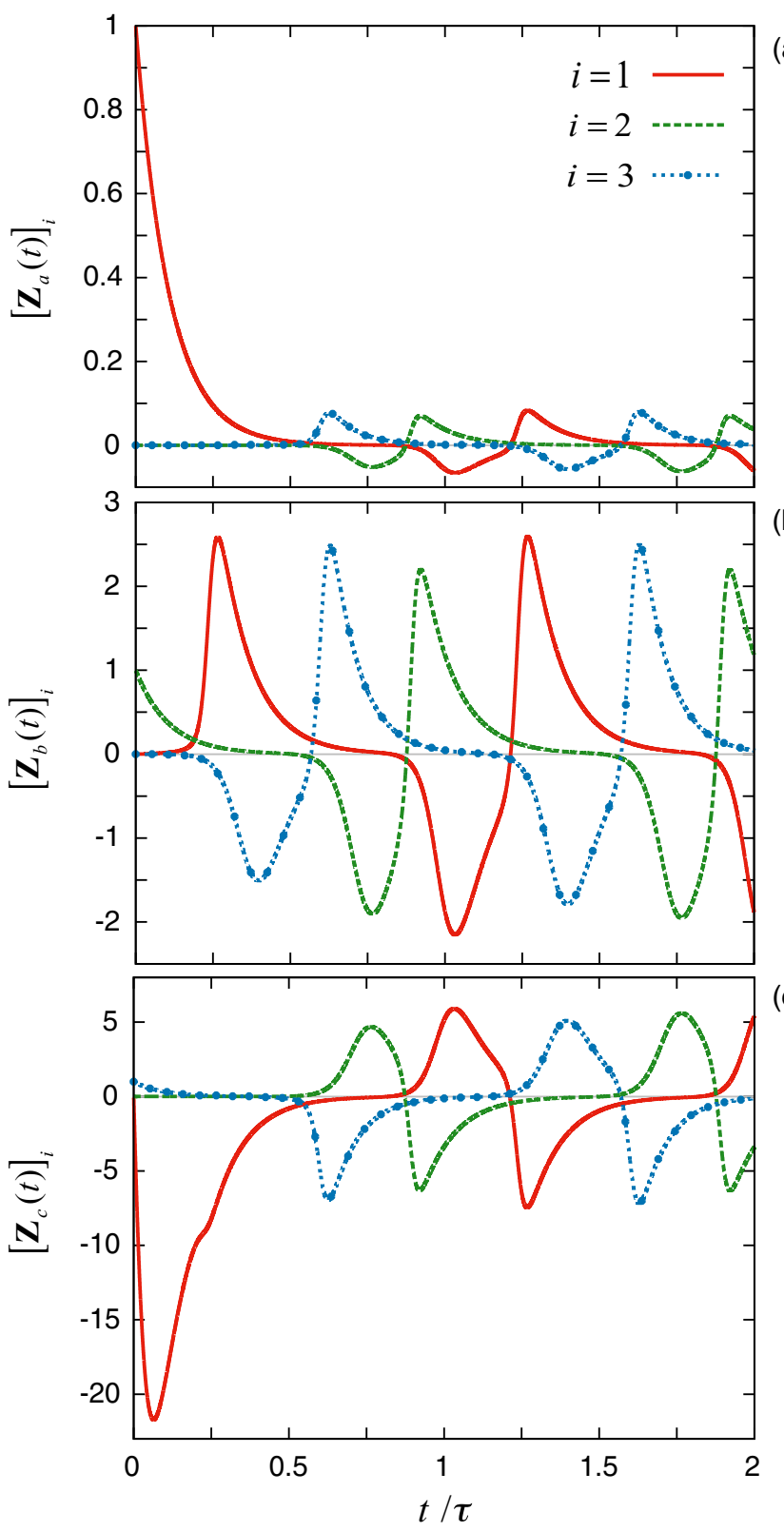

FIG. 6. The fundamental solutions of Eq. (9)- $\boldsymbol{z}_{a}(t), \boldsymbol{z}_{b}(t)$, and $z_{c}(t)$ - for the genetic repressilator model.
At sufficiently weak noise, the group of events corresponding to oscillation cycles is always well separated from the other three groups. We do not consider in this study the situation at so high noise levels that the groups begin to overlap. If this takes place, oscillations cannot be any longer distinguished from pure fluctuation effects.

\section{APPENDIX B}

We present explicit expressions for the Floquet properties in the considered genetic network model with three genes and the Hill coefficient $n=3$. The vector $\boldsymbol{f}[\boldsymbol{x}(t)]$ and matrix $\boldsymbol{G}(t)$ are

$$
\begin{gathered}
f_{i}[\boldsymbol{x}(t)]=\frac{1}{1+\kappa^{3} x_{\bmod (i+2,3)}^{3}}-a_{i} x_{i}, \\
G_{i i}[\boldsymbol{x}(t)]=\left(\frac{1+2 b}{1+\kappa^{3} x_{\bmod (i+2,3)}^{3}}+a_{i} x_{i}\right)^{\frac{1}{2}},
\end{gathered}
$$

and $G_{i j}[\boldsymbol{x}(t)]=0$ for $i \neq j$, where $\bmod (i, 3)$ represents the remainder of $i$ divided by 3 . Then the elements of the Jacobian matrix $\boldsymbol{\Gamma}(t)$ are given by

$$
\begin{gathered}
\Gamma_{i, i}(t)=-a_{i}, \\
\Gamma_{i, \bmod (i+1,3)}(t)=0,
\end{gathered}
$$

and

$$
\Gamma_{i, \bmod (i+2,3)}(t)=\frac{-3 \kappa^{3} p_{i+2}(t)^{2}}{\left[1+\kappa^{3} p_{i+2}(t)^{3}\right]^{2}} .
$$

The fundamental solutions of Eq. (9)- $z_{a}(t), z_{b}(t)$, and $z_{c}(t)-$ are shown in Fig. 6.

The matrix $\boldsymbol{B}$ for the system with the parameters and initial conditions given in $\mathrm{Sec}$. IV is

$$
\boldsymbol{B}=\left(\begin{array}{ccc}
-2.0244 & -0.97113 & -59.624 \\
0.018852 & 0.095496 & -1.7454 \\
-0.015951 & 0.022490 & -1.2711
\end{array}\right)
$$

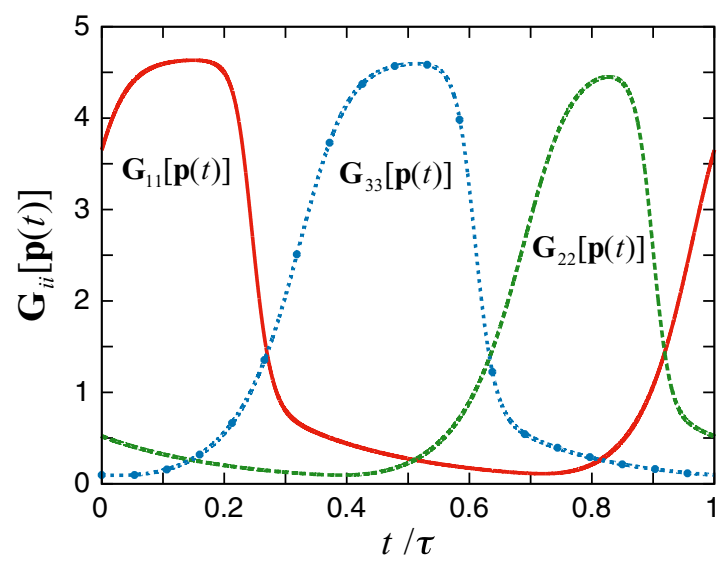

FIG. 7. The diagonal elements of the matrix $\boldsymbol{G}[\boldsymbol{p}(t)]$ yielding the intensity of internal noise for each gene. We can see that the trough of $\boldsymbol{G}_{11}[\boldsymbol{p}(t)]$ corresponding to the minimum internal noise for gene No. 1 clearly differs from checkpoint A, corresponding to the best single-cycle precision. 
The eigenvalues of matrix $\boldsymbol{B}$ are $\lambda_{0}=0, \lambda_{1}=-0.5067$, and $\lambda_{2}=-2.6932$. The right eigenvectors are ${ }^{t} \boldsymbol{\phi}_{0}=$ $(-0.8460,0.5328,0.0200),{ }^{t} \boldsymbol{\phi}_{1}=(-0.9947,0.0998,0.0237)$, and ${ }^{t} \boldsymbol{\phi}_{2}=(0.9999,0.0003,0.0112)$. The left eigenvectors are ${ }^{t} \boldsymbol{\psi}_{0}=(-0.0105,-0.3288,0.9443)$, ${ }^{t} \boldsymbol{\psi}_{1}=(0.0112,0.0553,-0.9984), \quad$ and ${ }^{t} \boldsymbol{\psi}_{2}=$ $(0.0238,0.0002,0.9997)$. The elements of the matrix $\boldsymbol{G}[\boldsymbol{p}(t)]$ are displayed graphically in Fig. 7 .
[1] Y. Kuramoto, Chemical Oscillations, Waves, and Turbulence (Springer, New York, 1984).

[2] A. T. Winfree, The Geometry of Biological Time, 2nd ed. (Springer, New York, 2001).

[3] S. M. Reppert and D. R. Weaver, Nature (London) 418, 935 (2002).

[4] L. Glass, Nature (London) 410, 277 (2001).

[5] K. T. Moortgat, T. H. Bullock, and T. J. Sejnowski, J. Neurophysiol. 83, 971 (2000).

[6] K. T. Moortgat, T. H. Bullock, and T. J. Sejnowski, J. Neurophysiol. 83, 984 (2000).

[7] H. Kori, Y. Kawamura, and N. Masuda, J. Theor. Biol. 297, 61 (2012).

[8] F. Mori and H. Kori, Phys. Rev. E 87, 030901 (2013).

[9] M. B. Elowitz and S. Leibler, Nature (London) 403, 335 (2000).

[10] J. Garcia-Ojalvo, M. B. Elowitz, and S. H. Strogatz, Proc. Natl. Acad. Sci. USA 101, 10955 (2004).

[11] H. R. Ueda, S. Hayashi, W. Chen, M. Sano, M. Machida, Y. Shigeyoshi, M. Iino, and S. Hashimoto, Nat. Gen. 37, 187 (2005).

[12] H. Ukai and H. R. Ueda, Annu. Rev. Physiol. 72, 579 (2010).
[13] Y. Kobayashi, T. Shibata, Y. Kuramoto, and A. S. Mikhailov, Eur. Phys. J. B 76, 167 (2010).

[14] Y. Kobayashi, T. Shibata, Y. Kuramoto, and A. S. Mikhailov, Phys. Rev. E 83, 060901 (2011).

[15] S. A. Stamper, M. S. Madhav, N. J. Cowan, and E. S. Fortune, J. Exp. Biol. 215, 4196 (2012).

[16] A. Mikhailov, L. Schimansky-Geier, and W. Ebeling, Phys. Lett. A 96, 453 (1983).

[17] Y. Kawamura, H. Nakao, K. Arai, H. Kori, and Y. Kuramoto, Phys. Rev. Lett. 101, 024101 (2008).

[18] J.-n. Teramae, H. Nakao, and G. B. Ermentrout, Phys. Rev. Lett. 102, 194102 (2009).

[19] D. S. Goldobin, J.-n. Teramae, H. Nakao, and G. B. Ermentrout, Phys. Rev. Lett. 105, 154101 (2010).

[20] H. Nakao, J.-n. Teramae, D. S. Goldobin, and Y. Kuramoto, Chaos 20, 033126 (2010).

[21] E. A. Coddington and R. Carlson, Linear Ordinary Differential Equations (SIAM, Philadelphia, 1997).

[22] K. Yoshimura and K. Arai, Phys. Rev. Lett. 101, 154101 (2008).

[23] R. F. Galán, Phys. Rev. E 80, 036113 (2009).

[24] T. Shibata, Phys. Rev. E 67, 061906 (2003). 